\title{
A M/M/1 Queueing Network with Classical Retrial Policy
}

\author{
${ }^{1}$ S. Shanmugasundaram, ${ }^{2}$ S. Vanitha \\ ${ }^{1}$ Assistant Professor of Mathematics, \\ Government Arts College (Autonomous), \\ Salem-636007, Tamil Nadu, India \\ E-mail: sundaramsss@hotmail.com \\ ${ }^{2}$ Ph.D. Research Scholar (Part-time), \\ Government Arts college (Autonomous), \\ Salem-636007, Tamil Nadu, India. \\ Email: vanitharamesh87@yahoo.co.in
}

Article History: Received: 11 January 2021; Accepted: 27 February 2021; Published online: 5 April 2021

\begin{abstract}
In this paper we study the M/M/1 queueing model with retrial on network. We derive the steady state probability of customers in the network, the average number of customers in the all the three nodes in the system, the queue length, system length using little's formula. The particular case is derived (no retrial). The numerical examples are given to test the correctness of the model.
\end{abstract}

Keywords: Queueing network, Retrial, Performance measures.

\section{Introduction}

A queue is a waiting line of people or things to be handled in a sequential order. A.K. Erlang [3] a Danish engineer who is called the father of queueing theory has published his articles relating to the study of congestion in telephone traffic in 1909.

Queueing network can be described as a group of nodes, where each node represents a service facility. The Queueing networks were first introduced by James. R. Jackson [7] in 1957. The most significant contribution in queueing network is Jackson's network [6]. Queueing network models have various applications in many areas, such as service centers, computer networks, communication networks, production and flexible manufacturing systems, airport terminals and healthcare systems etc. Queueing networks can be classified as open, closed and mixed networks. Open networks receive customers from an external source and send them to an external destination. Closed networks have a fixed population that moves between the queues but never leaves the system. Mixed networks open for some workloads and closed for others.

Queueing theory in which arriving customers who find the server occupied may retry for service after a period of time are called retrial queue or queues with repeated orders. Between retrials, the blocked customer join the pool of customers called 'orbit'. The concept of retrial queues was first introduced by Kosten [8] in 1947.Cohen [2] analyzed the basic problems of telephone traffic theory. Retrial queues can be classified as Classical retrial policy and Constant retrial policy. In Classical retrial policy the customers in the orbit act independently to each other, thus the retrial rate depends on the number of customers in the orbit. In constant retrial policy the repeated customers form a queue in orbit and only the customer at the head of the orbit can request a service after a random retrial time. Retrial queues are used as mathematical models of several computer systems, telecommunication networks, call centers, cellular networks, restaurant reservations, healthcare systems etc.

A two class retrial system with batch arrivals and classical retrial scheme was introduced by Kulkarni [9]. Falin [4] has analyzed an M/M/1 retrail queue without feedback. Fayolle [5] has investigated a M/M/1 retrial queue with constant retrial policy. Santhakumaran and Shanmugasundaram [12] have discussed a single server retrial queue with feedback. Atencia and Moreno [1] have focused on a single server retrial queue with general retrial times. Phung-Duc [11] has presented a survey on theory and applications of retrial queueing models. Sreekala and Manoharan [14] have focused on a queueing network model with feedback Tao Li et al [15] have proposed a $\mathrm{M} / \mathrm{M} / 1$ retrail queue with working vacation and classical retrial policy. Mohamed Boualem [10] et. al. have analyzed a single server feedback retrial queue. Shanmugasundaram and Vanitha [13] have analyzed an open queueing network system in healthcare .In this paper we consider an open queueing network with classical retrial policy 


\section{Description of the Model}

We consider an open queueing network consisting of three single nodes with retrial. External customers arrive to the system according to a Poisson process with rate $\lambda$ and get service with an exponential distribution. If the server is idle upon an arrival, service of an arriving customer at node 1 starts instantaneously. Otherwise, the arriving external customers either with probability $s$ enters the non retrial group or with probability $1-s$ joins the waiting space (orbit) where they waits to be served. If the server is occupied, customers are forced to wait in the orbit of infinite size and repeat their demand after an exponential time with parameter $\alpha$. The retrial rate for the customers from the orbit to the node 1 is $n \alpha$ where $n$ is the number of customers in the orbit. After getting the service in the first node they can either go to node 2 or node 3 with probability $p$ and with probability $1-p$ respectively. After getting the service at node 2 the customers can either leave the system with probability $q$ or they go to node 3 with probability $1-q$. After completing the service at node 3 the customer leave the system. Each node follows an M/M/1 schedule. The service rates for node 1 , node 2 and node 3 are with service rates $\mu_{1}, \mu_{2}$, and $\mu_{3}$ respectively. Fig. 1 represents the system.

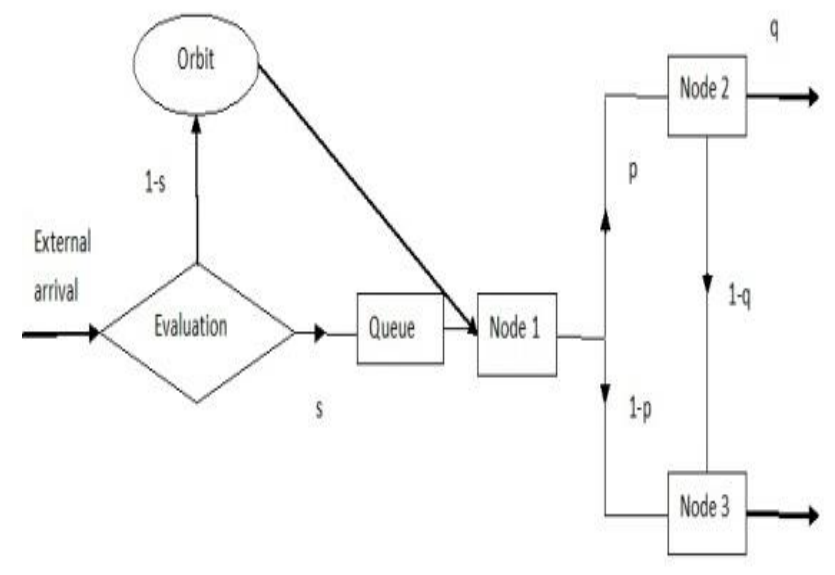

\section{Balance Equations}

Fig. 1

We define $\lambda_{i}$ where $(\mathrm{i}=1,2,3)$ is the arrival rate to each nodes. The balance equations for this model are obtained as given below:

$\lambda_{1}=\lambda s+n \alpha$

$\lambda_{2} \quad=\lambda_{1} p=(\lambda s+n \alpha) p$

$\lambda_{3}=\lambda_{1}(1-p)+\lambda_{2}(1-q)=(\lambda s+n \alpha)(1-p q)(3)$ If $n_{1}, n_{2}, n_{3}$ are the number of customers at each nodes then using Jackson network the steady state solution is denoted by $P\left(n_{1}, n_{2}, n_{3}\right)$.

The steady state probability for $n_{1}, n_{2}, n_{3}$ customers at the three nodes respectively is

$$
\begin{aligned}
& P\left(n_{1}, n_{2}, n_{3}\right)=\left(1-\rho_{1}\right) \rho_{1}^{n_{1}}\left(1-\rho_{2}\right) \rho_{2}^{n_{2}}\left(1-\rho_{3}\right) \rho_{3}^{n_{3}}(4) \text { where, } \rho_{1}=\frac{\lambda_{1}}{\mu_{1}}, \rho_{2}=\frac{\lambda_{2}}{\mu_{2}}, \rho_{3}=\frac{\lambda_{3}}{\mu_{3}} \\
& P\left(n_{1}, n_{2}, n_{3}\right)=\left(1-\frac{\lambda_{1}}{\mu_{1}}\right)\left(\frac{\lambda_{1}}{\mu_{1}}\right)^{n_{1}}\left(1-\frac{\lambda_{2}}{\mu_{2}}\right)\left(\frac{\lambda_{2}}{\mu_{2}}\right)^{n_{2}}\left(1-\frac{\lambda_{3}}{\mu_{3}}\right)\left(\frac{\lambda_{3}}{\mu_{3}}\right)^{n_{3}}=\left(1-\frac{\lambda s+n \alpha}{\mu_{1}}\right)\left(\frac{\lambda s+n \alpha}{\mu_{1}}\right)^{n_{1}}\left(1-\frac{(\lambda s+n \alpha) p}{\mu_{2}}\right)\left(\frac{(\lambda s+n \alpha) p}{\mu_{2}}\right)^{n_{2}} \\
& \left(1-\frac{(\lambda s+n \alpha)(1-p q)}{\mu_{3}}\right)\left(\frac{(\lambda s+n \alpha)(1-p q)}{\mu_{3}}\right)^{n_{3}}
\end{aligned}
$$

$N_{i}(i=1,2,3)$ be the number of customers in the node. We have,

The number of customers in the first node $N_{1}$ is,

$$
N_{1}=\frac{\rho_{1}}{1-\rho_{1}}=\frac{\lambda_{1}}{\mu_{1}-\lambda_{1}}=\frac{\lambda s+n \alpha}{\mu_{1}-(\lambda s+n \alpha)}
$$

(6) The number of customers in the second node is, 


$$
\begin{aligned}
& N_{2} \quad=\frac{\rho_{2}}{1-\rho_{2}}=\frac{\lambda_{2}}{\mu_{2}-\lambda_{2}}=\frac{(\lambda s+n \alpha) p}{\mu_{2}-(\lambda s+n \alpha) p} \\
& N_{3} \quad=\frac{\rho_{3}}{1-\rho_{3}}=\frac{\lambda_{3}}{\mu_{3}-\lambda_{3}}=\frac{(\lambda s+n \alpha)(1-p q)}{\mu_{3}-(\lambda s+n \alpha)(1-p q)} \\
& L_{s_{1}}=N_{1}=\frac{\lambda s+n \alpha}{\mu_{1}-(\lambda s+n \alpha)}
\end{aligned}
$$

(7) The number of customers in the third node is,

(8) The average number of customers in node 1 is,

Average waiting time of a customer in node 1 is,

$$
\begin{aligned}
& W_{s_{1}}=\frac{L_{s_{1}}}{\lambda_{1}}=\frac{1}{\mu_{1}-(\lambda s+n \alpha)} \\
& L_{q_{1}}=L_{s_{1}}-\frac{\lambda_{1}}{\mu_{1}}=\frac{\lambda s+n \alpha}{\mu_{1}-(\lambda s+n \alpha)}-\frac{\lambda s+n \alpha}{\mu_{1}}
\end{aligned}
$$

is

$$
\begin{aligned}
& W_{q_{1}}=\frac{L_{q_{1}}}{\lambda_{1}}=\frac{1}{\mu_{1}-(\lambda s+n \alpha)}-\frac{1}{\mu_{1}} \\
& L_{s_{2}}=N_{2}=\frac{(\lambda s+n \alpha) p}{\mu_{2}-(\lambda s+n \alpha) p} \\
& L_{s_{3}}=N_{3}=\frac{(\lambda s+n \alpha)(1-p q)}{\mu_{3}-(\lambda s+n \alpha)(1-p q)}
\end{aligned}
$$

(12) The average number of customers in node 2 is,

(13) The average number of customers in node 3 is

The remaining parameters are calculated using

little's formula.

Average waiting time of a customer in node 2 is,

$$
\begin{aligned}
& W_{s_{2}}=\frac{L_{s_{2}}}{\lambda_{2}}=\frac{1}{\mu_{2}-(\lambda s+n \alpha) p} \\
& L_{q_{2}}=L_{s_{2}}-\frac{\lambda_{2}}{\mu_{2}}=\frac{(\lambda s+n \alpha) p}{\mu_{2}-(\lambda s+n \alpha) p}-\frac{(\lambda s+n \alpha) p}{\mu_{2}}
\end{aligned}
$$

is

$$
W_{q_{2}}=\frac{L_{q_{2}}}{\lambda_{2}}=\frac{1}{\mu_{2}-(\lambda s+n \alpha) p}-\frac{1}{\mu_{2}}
$$

is

$$
\begin{aligned}
& W_{s_{3}}=\frac{L_{s_{3}}}{\lambda_{3}}=\frac{1}{\mu_{3}-(\lambda s+n \alpha)(1-p q)} \quad(18) \text { The average queue length of node } 3 \text { is } \\
& L_{q_{3}} \quad=L_{s_{3}}-\frac{\lambda_{3}}{\mu_{3}} \\
& =\frac{(\lambda s+n \alpha)(1-p q)}{\mu_{3}-(\lambda s+n \alpha)(1-p q)}-\frac{(\lambda s+n \alpha)(1-p q)}{\mu_{3}} \quad(19) \text { Average waiting time of a customer in node } 3 \text { is } \\
& W_{q_{3}}=\frac{L_{q_{3}}}{\lambda_{3}}=\frac{1}{\mu_{3}-(\lambda s+n \alpha)(1-p q)}-\frac{1}{\mu_{3}}(20) \text { Average number of customers in the overall system, }
\end{aligned}
$$




$$
L_{s} \quad=N_{1}+N_{2}+N_{3} \quad=\frac{\lambda s+n \alpha}{\mu_{1}-(\lambda s+n \alpha)}+\frac{(\lambda s+n \alpha) p}{\mu_{2}-(\lambda s+n \alpha) p}+\frac{(\lambda s+n \alpha)(1-p q)}{\mu_{3}-(\lambda s+n \alpha)(1-p q)} \quad \text { (21) Average }
$$

waiting time of a customer in the system,

$W_{s} \quad=\frac{L_{s}}{\lambda} \quad($ By little's formula $)$

$=\frac{1}{\lambda}\left[\frac{\lambda s+n \alpha}{\mu_{1}-(\lambda s+n \alpha)}+\frac{(\lambda s+n \alpha) p}{\mu_{2}-(\lambda s+n \alpha) p}+\frac{(\lambda s+n \alpha)(1-p q)}{\mu_{3}-(\lambda s+n \alpha)(1-p q)}\right](22)$ Average number of customers in all the three queues

$L_{q} \quad=L_{s}-\frac{\lambda}{\mu} \quad($ By little's formula $)$

$=\frac{\lambda s+n \alpha}{\mu_{1}-(\lambda s+n \alpha)}+\frac{(\lambda s+n \alpha) p}{\mu_{2}-(\lambda s+n \alpha) p}+\frac{(\lambda s+n \alpha)(1-p q)}{\mu_{3}-(\lambda s+n \alpha)(1-p q)}-\frac{\lambda}{\mu}(23)$ Average waiting time of a customer in all the three queues

$W_{q} \quad=\frac{L_{q}}{\lambda} \quad$ (By little's formula $)$

$$
=\frac{1}{\lambda}\left(\frac{\lambda s+n \alpha}{\mu_{1}-(\lambda s+n \alpha)}+\frac{(\lambda s+n \alpha) p}{\mu_{2}-(\lambda s+n \alpha) p}+\frac{(\lambda s+n \alpha)(1-p q)}{\mu_{3}-(\lambda s+n \alpha)(1-p q)}\right)-\frac{1}{\mu}(24) \text { IV Particular Case }
$$

If there is no retrial queue then $\alpha \rightarrow 0$ and $s \rightarrow 1$ then we get,

$$
\begin{aligned}
& \lambda_{1}=\lambda \quad, \lambda_{2}=\lambda p, \lambda_{3}=\lambda(1-p q) \\
& L_{s} \quad=\frac{\lambda}{\mu_{1}-\lambda}+\frac{\lambda p}{\mu_{2}-\lambda p}+\frac{\lambda(1-p q)}{\mu_{3}-\lambda(1-p q)} \\
& W_{s} \quad=\frac{1}{\mu_{1}-\lambda}+\frac{p}{\mu_{2}-\lambda p}+\frac{1-p q}{\mu_{3}-\lambda(1-p q)} \\
& L_{q} \quad=\frac{\lambda}{\mu_{1}-\lambda}+\frac{\lambda p}{\mu_{2}-\lambda p}+\frac{\lambda(1-p q)}{\mu_{3}-\lambda(1-p q)}-\frac{\lambda}{\mu} \\
& W_{q} \quad=\frac{1}{\mu_{1}-\lambda}+\frac{p}{\mu_{2}-\lambda p}+\frac{1-p q}{\mu_{3}-\lambda(1-p q)}-\frac{1}{\mu}
\end{aligned}
$$

(29) In this case, the results are coincide with

Shanmugasundaram and Vanitha[13].

\section{Numerical Examples}

In this section we investigate the steady state solution and the performance measures.

For $\lambda=0.7, \alpha=0.5, \mu_{1}=5.5, \mu_{2}=6, \mu_{3}=6.5$,

$$
p=0.3, q=0.4, s=0.2, n=5 .
$$

The steady state probability values for $n_{1}, n_{2}, n_{3}$ customers at the three nodes respectively are given in Table 1 .

\section{Calculation for the system:}

For the arrival rate $\lambda$ from 0.7 to 1.1 and for $\mathrm{n}$ (the number of customers in the orbit) from 1 to 10 , the average number of customers in all the three queues is calculated in Table 2. From Fig. 2 and Fig. 3 it is clear that as the arrival rate increases for various values of $\mathrm{n}$, the average number of customers in all the three queues increases.

Table 1

\begin{tabular}{|c|c|c|c|c|c|c|c|}
\hline$\left(n_{1}, n_{2}, n_{3}\right)$ & $P\left(n_{1}, n_{2}, n_{3}\right)$ & $\left(n_{1}, n_{2}, n_{3}\right)$ & $P\left(n_{1}, n_{2}, n_{3}\right)$ & $\left(n_{1}, n_{2}, n_{3}\right)$ & $P\left(n_{1}, n_{2}, n_{3}\right)$ & $\left(n_{1}, n_{2}, n_{3}\right)$ & $P\left(n_{1}, n_{2}, n_{3}\right)$ \\
\hline 000 & $2.90 \mathrm{E}-01$ & 100 & $1.39 \mathrm{E}-01$ & 200 & $6.68 \mathrm{E}-02$ & 300 & $3.21 \mathrm{E}-02$ \\
\hline 001 & $1.04 \mathrm{E}-01$ & 101 & $4.98 \mathrm{E}-02$ & 201 & $2.39 \mathrm{E}-02$ & 301 & $1.15 \mathrm{E}-02$ \\
\hline 002 & $3.71 \mathrm{E}-02$ & 102 & $1.78 \mathrm{E}-02$ & 202 & $8.54 \mathrm{E}-03$ & 302 & $4.10 \mathrm{E}-03$ \\
\hline
\end{tabular}


${ }^{1}$ S. Shanmugasundaram, ${ }^{2} \mathrm{~S}$. Vanitha

\begin{tabular}{|c|c|c|c|c|c|c|c|}
\hline 003 & $1.32 \mathrm{E}-02$ & 103 & $6.36 \mathrm{E}-03$ & 203 & $3.05 \mathrm{E}-03$ & 303 & $1.46 \mathrm{E}-03$ \\
\hline 010 & $3.83 \mathrm{E}-02$ & 110 & $1.84 \mathrm{E}-02$ & 210 & $8.82 \mathrm{E}-03$ & 310 & $4.23 \mathrm{E}-03$ \\
\hline $0 \begin{array}{lll}0 & 11\end{array}$ & $1.37 \mathrm{E}-02$ & 111 & $6.57 \mathrm{E}-03$ & 211 & $3.15 \mathrm{E}-03$ & 311 & $1.51 \mathrm{E}-03$ \\
\hline 012 & $4.89 \mathrm{E}-03$ & 112 & $2.35 \mathrm{E}-03$ & 212 & $1.13 \mathrm{E}-03$ & 312 & $5.41 \mathrm{E}-04$ \\
\hline 013 & $1.75 \mathrm{E}-03$ & 113 & 8.39E-04 & 213 & $4.03 \mathrm{E}-04$ & 313 & $1.93 \mathrm{E}-04$ \\
\hline 020 & $5.05 \mathrm{E}-03$ & 120 & $2.43 \mathrm{E}-03$ & 220 & $1.16 \mathrm{E}-03$ & 320 & $5.59 \mathrm{E}-04$ \\
\hline 021 & $1.81 \mathrm{E}-03$ & 121 & $8.67 \mathrm{E}-04$ & 221 & $4.16 \mathrm{E}-04$ & 321 & $2.00 \mathrm{E}-04$ \\
\hline 022 & $6.46 \mathrm{E}-04$ & 122 & $3.10 \mathrm{E}-04$ & 222 & $1.49 \mathrm{E}-04$ & 322 & $7.14 \mathrm{E}-05$ \\
\hline 023 & $2.31 \mathrm{E}-04$ & 123 & $1.11 \mathrm{E}-04$ & 223 & $5.32 \mathrm{E}-05$ & 323 & $2.55 \mathrm{E}-05$ \\
\hline 030 & $6.67 \mathrm{E}-04$ & 130 & $3.20 \mathrm{E}-04$ & 230 & $1.54 \mathrm{E}-04$ & 330 & $7.38 \mathrm{E}-05$ \\
\hline 031 & $2.38 \mathrm{E}-04$ & 131 & $1.14 \mathrm{E}-04$ & 231 & $5.49 \mathrm{E}-05$ & 331 & $2.64 \mathrm{E}-05$ \\
\hline 032 & $8.52 \mathrm{E}-05$ & 132 & $4.09 \mathrm{E}-05$ & 232 & $1.96 \mathrm{E}-05$ & 332 & $9.42 \mathrm{E}-06$ \\
\hline 033 & $7.48 \mathrm{E}-06$ & 133 & $1.97 \mathrm{E}-06$ & 233 & $5.21 \mathrm{E}-07$ & 333 & $1.38 \mathrm{E}-07$ \\
\hline
\end{tabular}

Table 2

\begin{tabular}{|c|c|c|c|c|c|}
\hline$n / \lambda$ & 0.7 & 0.8 & 0.9 & 1 & 1.1 \\
\hline 1 & 0.1429 & 0.1353 & 0.1277 & 0.1201 & 0.1126 \\
\hline 2 & 0.3878 & 0.3818 & 0.3760 & 0.3702 & 0.3645 \\
\hline 3 & 0.6829 & 0.6794 & 0.6759 & 0.6726 & 0.6693 \\
\hline 4 & 1.0480 & 1.0477 & 1.0477 & 1.0478 & 1.0481 \\
\hline 5 & 1.5147 & 1.5195 & 1.5245 & 1.5298 & 1.5354 \\
\hline 6 & 2.1395 & 2.1524 & 2.1657 & 2.1795 & 2.1937 \\
\hline 7 & 3.0344 & 3.0620 & 3.0904 & 3.1196 & 3.1497 \\
\hline 8 & 4.4638 & 4.5231 & 4.5845 & 4.6480 & 4.7137 \\
\hline 9 & 7.2703 & $7.4273 \mathrm{~s}$ & 7.5921 & 7.7651 & 7.9471 \\
\hline 10 & 16.7951 & 17.7085 & 18.7348 & 19.8964 & 21.2223 \\
\hline
\end{tabular}

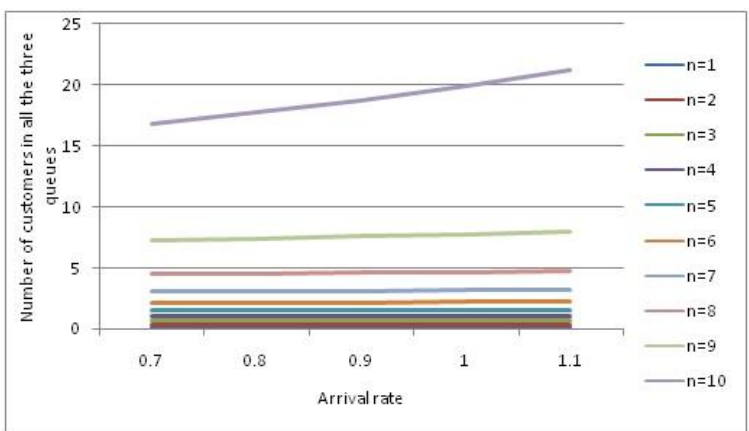

Fig. 2

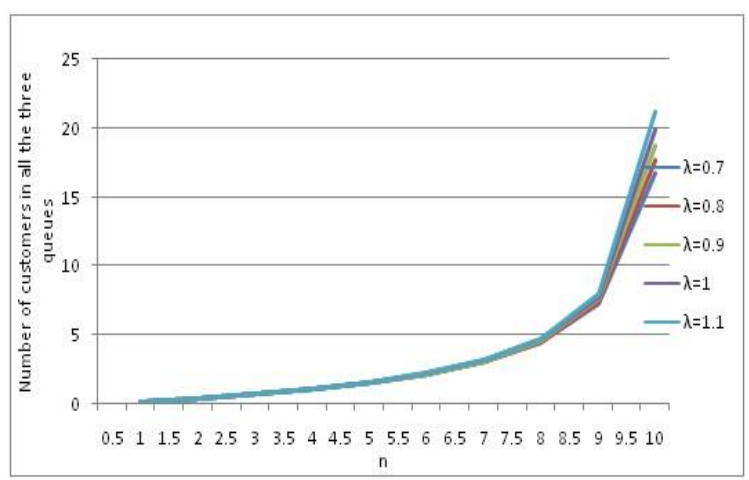

Fig. 3

For the arrival rate $\lambda$ from 0.7 to 1.1 and for $\mathrm{n}$ (the number of customers in the orbit) from 1 to 10 , the waiting time of a customer in all the three queues is calculated in Table 3. From Fig. 4 and Fig. 5 it is clear that as 
the arrival rate increases, the waiting time of a customer in all the three queues decreases and as the $\mathrm{n}$ value increases, the waiting time of a customer in all the three queues increases.

Table 3

\begin{tabular}{|c|c|c|c|c|c|}
\hline$n / \lambda$ & 0.7 & 0.8 & 0.9 & 1 & 1.1 \\
\hline 1 & 0.2042 & 0.1691 & 0.1419 & 0.1201 & 0.1024 \\
\hline 2 & 0.5539 & 0.4773 & 0.4177 & 0.3702 & 0.3314 \\
\hline 3 & 0.9756 & 0.8492 & 0.7510 & 0.6726 & 0.6085 \\
\hline 4 & 1.4971 & 1.3097 & 1.1641 & 1.0478 & 0.9528 \\
\hline 5 & 2.1639 & 1.8994 & 1.6939 & 1.5298 & 1.3958 \\
\hline 6 & 3.0565 & 2.6905 & 2.4064 & 2.1795 & 1.9943 \\
\hline 7 & 4.3349 & 3.8275 & 3.4337 & 3.1196 & 2.8633 \\
\hline 8 & 6.3768 & 5.6539 & 5.0939 & 4.6480 & 4.2852 \\
\hline 9 & 10.3861 & 9.2841 & 8.4356 & 7.7651 & 7.2246 \\
\hline 10 & 23.9931 & 22.1357 & 20.8164 & 19.8964 & 19.2930 \\
\hline
\end{tabular}

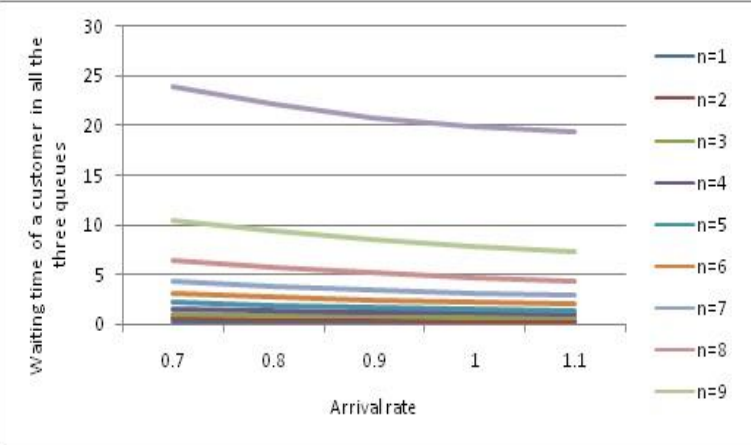

Fig.4

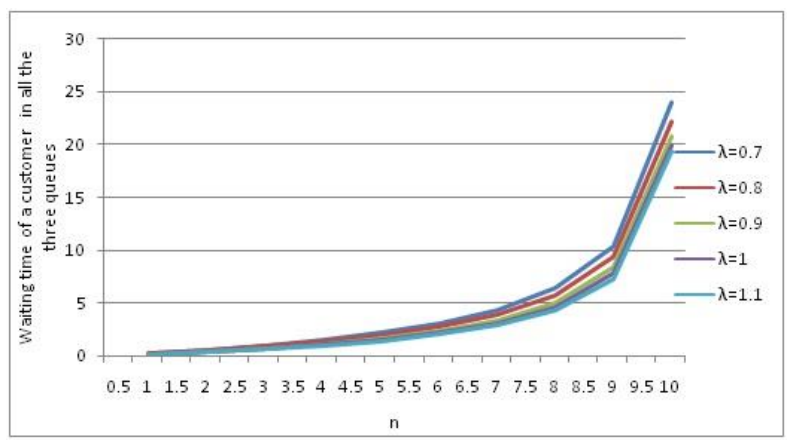

Fig. 5

For the service rate from 5.5 to 5.9 and for $\mathrm{n}$ (the number of customers in the orbit) from 1 to 10 , the number of customers in the system is calculated in Table 4. From Fig.6 and Fig. 7 it is clear that as the service rate increases, the number of customers in the system decreases and as the $\mathrm{n}$ value increases, the number of customers in the system increases.

Table 4

\begin{tabular}{|c|c|c|c|c|c|}
\hline $\begin{array}{l}n \\
/ \mu_{1}\end{array}$ & 5.5 & 5.6 & 5.7 & 5.8 & 5.9 \\
\hline 1 & 0.2596 & 0.2570 & 0.2544 & 0.2520 & 0.2496 \\
\hline 2 & 0.5044 & 0.4986 & 0.4930 & 0.4876 & 0.4824 \\
\hline 3 & 0.7996 & 0.7889 & 0.7787 & 0.7690 & 0.7597 \\
\hline 4 & 1.1646 & 1.1462 & 1.1288 & 1.1124 & 1.0969 \\
\hline 5 & 1.6314 & 1.6002 & 1.5710 & 1.5437 & 1.5181 \\
\hline
\end{tabular}




\begin{tabular}{|c|c|c|c|c|c|}
\hline 6 & 2.2562 & 2.2021 & 2.1523 & 2.1061 & 2.0634 \\
\hline 7 & 3.1511 & 3.0512 & 2.9611 & 2.8793 & 2.8047 \\
\hline 8 & 4.5804 & 4.3719 & 4.1902 & 4.0303 & 3.8886 \\
\hline 9 & 7.3869 & 6.8249 & 6.3689 & 5.9916 & 5.6741 \\
\hline 10 & 16.9118 & 13.8080 & 11.8126 & 10.4219 & 9.3972 \\
\hline
\end{tabular}

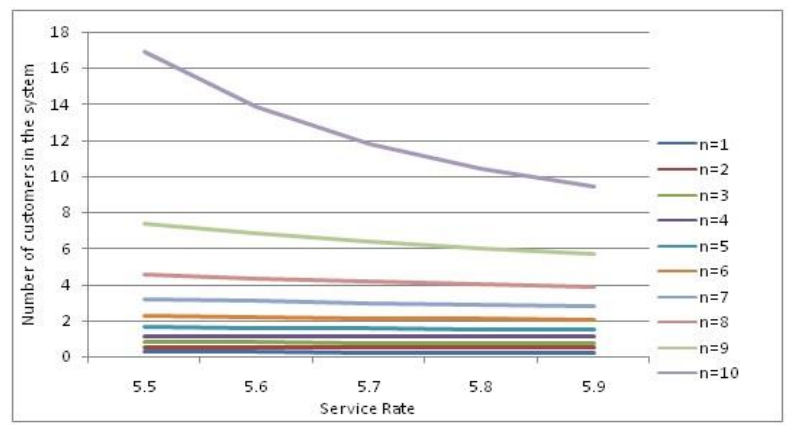

Fig.6

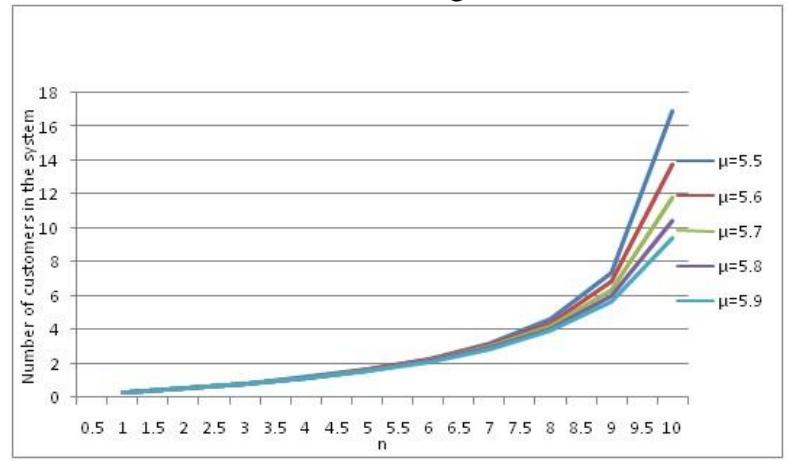

Fig. 7

For the service rate from 5.5 to 5.9 and for $\mathrm{n}$ (the number of customers in the orbit) from 1 to 10 , the waiting time of a customer in the system is calculated in Table 5. From Fig. 8 and Fig. 9 it is clear that as the service rate increases, the waiting time of a customer in the system decreases and as the $\mathrm{n}$ value increases, the waiting time of a customer in the system increases.

Table 5

\begin{tabular}{|c|c|c|c|c|c|}
\hline$n / \mu_{1}$ & 5.5 & 5.6 & 5.7 & 5.8 & 5.9 \\
\hline 1 & 0.3709 & 0.3671 & 0.3634 & 0.3599 & 0.3566 \\
\hline 2 & 0.7206 & 0.7122 & 0.7042 & 0.6966 & 0.6892 \\
\hline 3 & 1.1423 & 1.1269 & 1.1124 & 1.0985 & 1.0853 \\
\hline 4 & 1.6638 & 1.6375 & 1.6126 & 1.5892 & 1.5670 \\
\hline 5 & 2.3305 & 2.2860 & 2.2443 & 2.2053 & 2.1687 \\
\hline 6 & 3.2231 & 3.1459 & 3.0747 & 3.0088 & 2.9477 \\
\hline 7 & 4.5016 & 4.3589 & 4.2301 & 4.1133 & 4.0067 \\
\hline 8 & 6.5435 & 6.2456 & 5.9859 & 5.7575 & 5.5551 \\
\hline 9 & 10.5528 & 9.7499 & 9.0985 & 8.5594 & 8.1059 \\
\hline 10 & 24.1597 & 19.7256 & 16.8752 & 14.8885 & 13.4246 \\
\hline \multicolumn{5}{|c}{} \\
\hline
\end{tabular}




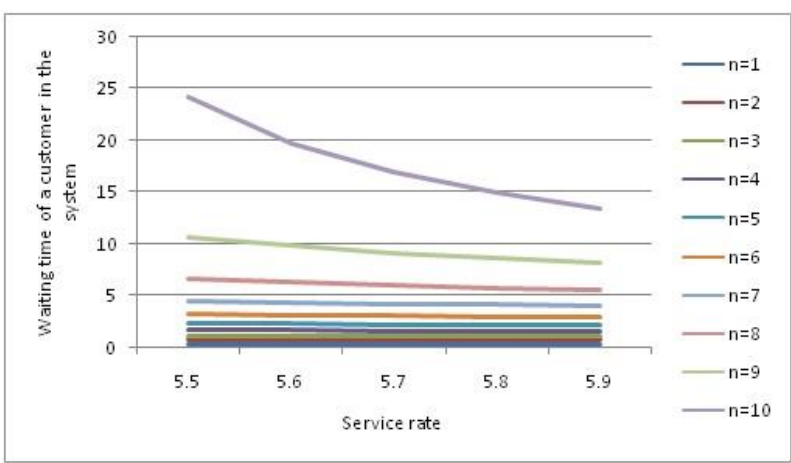

Fig. 8

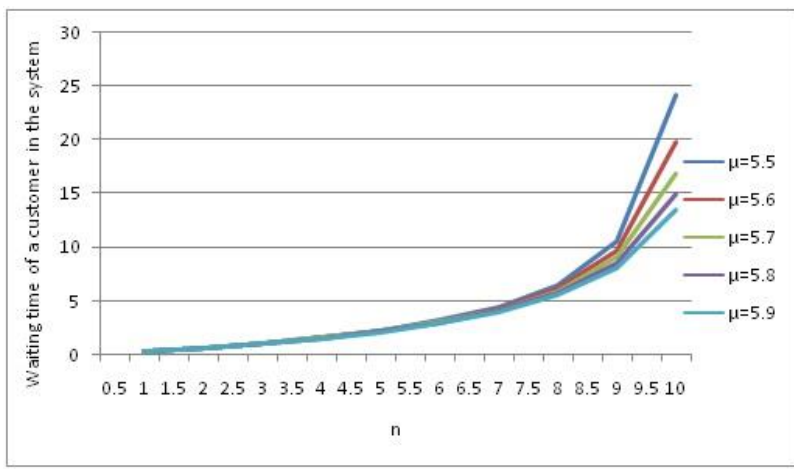

Fig. 9

\section{Conclusion}

Here we analyze the queueing network consists of three nodes with retrial policy. We derived the steady state probability of customers in the network, the average number of customers in the all the three nodes in the system, the queue length, system length using Little's formula. The numerical examples shows that as the arrival rate $(\lambda)$ increases for various $n$ values, the number of customers in all the three queues increases and the waiting time of a customer in all the three queues decreases. As the service rate increases, the number of customers in the system decreases and the waiting time of a customer in the system decrease. Also as the $\mathrm{n}$ (the number of customers in the orbit) value increases the average queue length and the waiting time of a customer in the queue for the system increases and the number of customers and the waiting time of a customer for the system all are increases. Also in the particular case if there is no retrial policy then the number of customers in the three nodes $\left(N_{1}, N_{2}, N_{3}\right)$ shows the correctness.

\section{REFERENCES}

1. Atencia I. and Moreno P., "A single server retrial queue with general retrial times and Bernoulli schedule", Appl.Math.Comput. 162, pp.855-880 2005.

2. Cohen J.W., "Basic problems of telephone traffic theory and the influence of repeated calls", Philips Telecommunication Review 18, pp. 49-100 1957.

3. Erlang A.K., "The theory of probabilities and telephone conversations", NytJindsskriff Math, B 20, pp.33-39 1909.

4. Falin G.I, A survey of retrial queues, Queueing system 7, pp.127-167 1990.

5. Fayolle G., "A simple telephone exchange with delayed feedbacks", International seminar on Teletraffic Analysis and Computer Performance Evaluation, North-Holland Publishing Co., Amsterdam, pp. 245-253 1986.

6. Jackson J.R., “Job shop-like queueing systems”, Management Science 10 pp.131-142 1963.

7. Jackson J.R.,"Networks of waiting lines", Operation Research 5(4) pp.518-521 1957.

8. Kosten L., "On the influence of repeated calls in the theory of probabilities of blocking", De Ingenieur 59, pp.1251947.

9. Kulkarni V.G.,“On queueing systems with retrials”, J.Appl.Prob.20, pp.380-389 1983. 
10. Mohamed Boualem, Natalia Djellab and DjamilAissani, "Stochastic Approximations and Monotonicity of a single server feedback retrial queue", Mathematical problems in Engineering, Hindawi Publishing Corporation 2012.

11. Phung-Duc T., Stochastic operations Research in Business and Industry, World scientific Publisher 2017.

12. Santhakumaran A. and Shanmugasundaram S., "A Single server retrial queue in Bernoulli schedule with feedback on non-retrial customers", Southeast Asian Bulletin of Mathematics", Vol.35, pp.305-317 2011.

13. Shanmugasundaram S. and Vanitha S., "An Application to Health Care System in (M/M/1) Queueing Model", Journal of Emerging Technologies and Innovative Research, Vol.6 Issue 6, pp.863-873 2019.

14. Sreekala M.S. and Manoharan M., "A queueing network model with feedback and its application in healthcare", International Journal of new technologies in science \& Engineering Vol.3 Issue 12, pp.78-89 2016.

15. Tao Li, Liyuan Zhang, and Shan Gao, "Performance of an M/M/1 Retrial Queue with Working Vacation Interruption and Classical Retrial Policy", Advances in Operation Research, Hindawi Publishing Corporation 2016. 